Pesq. Vet. Bras. 30(1):29-36, janeiro 2010

\title{
Avaliação de técnicas de biópsia hepática em ovinos ${ }^{1}$
}

\author{
Pedro B. Néspoli2*, Valentim A. Gheller ${ }^{3}$, Paulo V. Peixoto ${ }^{4}$, Ticiana N. França ${ }^{5}$, \\ Antônio U. Carvalho ${ }^{3}$, Douglas K. Godoy de Araújo ${ }^{6}$ e Christina Malm ${ }^{3}$
}

\begin{abstract}
Néspoli P.B, Gheller V.A., Peixoto P.V., França T.N., Carvalho A.U., Godoy de Araújo D.K. \& Malm C. 2010. [Evaluation of hepatic biopsy techniques in sheep.] Avaliação de técnicas de biópsia hepática em ovinos. Pesquisa Veterinária Brasileira 30(1):29-36. Departamento de Clínica Médica Veterinária, Universidade Federal de Mato Grosso, Av. Fernando Corrêa da Costa s/n, Coxipó, Cuiabá, MT 78060-900, Brazil. Email: nespoli@ufmt.br

Due to lack of information about the subject and lack of studies that confront the techniques of liver biopsy in sheep, a comparative study between eight biopsy techniques in this species was developed. In this study, eight crossbred (Santa Inês) ewe lambs $(17.95 \mathrm{~kg} \pm 2.71)$ were used in serial procedures for eight consecutive weeks. Liver samples obtained through six percutaneous techniques and two videolaparoscopic techniques were analyzed. The clinical aspects, clinical pathological findings, hepatic sample weights, histology quality, number of centrilobular veins and portal spaces, and the presence of artifacts in the histology slices were recorded. For the percutaneous techniques, two types of needles were used, modified Menghini (M) and semi-automatic tru-cut $(\mathrm{T})$. They were used in combinations with blind biopsies, guided by ultrasound (US) and monitored by video laparoscopy (VL) biopsies. In the videolaparoscopy procedures, Blakesley biopsy forceps and the method of liver fragment resection by VL were used. No relevant hematological or biochemical alterations were found and the clinical manifestations were slight and transitory. In a general way, the use of US and VL in percutaneous biopsies did not yield a significant improvement in weight and histology quality of the liver samples; however, the videolaparoscopic techniques allowed a wider access to the liver than the percutaneous techniques. The quality of the samples obtained through both $\mathrm{VL}$ techniques were equivalent; however, the resection technique enabled the collection of heavier samples, but determined the formation of perihepatic adherences. More difficulty in fragment retrieval, more fluctuation in weight and less quality were found in samples obtained with $\mathrm{M}$ needles when compared to $\mathrm{T}$ needles.
\end{abstract}

INDEX TERMS: Liver biopsy, videolaparoscopy, ultrasonography, sheep.

\footnotetext{
${ }^{1}$ Recebido em 13 de junho de 2009.

Aceito para publicação em 18 de agosto de 2009.

2 Departamento de Clínica Médica Veterinária, Faculdade Agronomia e Medicina Veterinária, Universidade Federal de Mato Grosso (UFMT), Av. Fernando Corrêa da Costa s/n, Coxipó, Cuiabá, MT 78060-900, Brasil. "Autor para correspondência: nespoli@ufmt.br

${ }^{3}$ Departamento de Clínica e Cirurgia Veterinárias, Escola de Veterinária, Universidade Federal de Minas Gerais (UFMG), Av. Antônio Carlos 6627, Cx. Postal 567, Belo Horizonte, MG 30123-970, Brasil.

${ }^{4}$ Departamento de Nutrição Animal e Pastagem, Instituto de Zootecnia, Universidade Federal Rural do Rio de Janeiro (UFRRJ), Seropédica, RJ 23890-000, Brasil.

${ }^{5}$ Departamento de Epidemiologia e Saúde Pública, Instituto de Veterinária, UFRRJ, Seropédica, RJ, Brasil.

${ }^{6}$ Graduação em Medicina Veterinária, Escola de Veterinária, UFMG, Belo Horizonte, MG, Brasil.
}

RESUMO.- Devido à carência de informações sobre o tema e à ausência de estudos que confrontem as técnicas de biópsia hepática em ovinos, foi desenvolvido um estudo comparativo entre oito técnicas de biópsia nesta espécie. Neste estudo foram utilizadas oito borregas $(17,95 \mathrm{~kg} \pm 2,71)$ mestiças (Santa Inês) em procedimentos seriados por oito semanas consecutivas e avaliados os aspectos clínicos, os achados de patologia clínica, o peso das amostras hepáticas, a qualidade histológica, o número de veias centrolobulares e espaços-porta e a presença de artefatos no corte histológico de amostras hepáticas obtidas através de seis técnicas percutâneas e duas técnicas videolaparoscópicas. Para as técnicas percutâneas empregaram-se dois tipos de agulhas, Men- 
ghini modificada (M) e Tru-cut semi-automática (T), combinadas com biópsias cegas, guiadas por ultra-sonografia (US) e monitoradas por videolaparoscopia (VL). No caso dos procedimentos vídeolaparoscópicos utilizaram-se pinça laparoscópica de biópsia Blakesley e método de ressecção de fragmento hepático por VL. Não foram observadas alterações hematológicas ou bioquímicas relevantes e as manifestações clínicas detectadas foram leves e transitórias. De uma forma geral, o uso da US e da VL nas biópsias percutâneas não resultou em acréscimo significativo do peso e da qualidade histológica das amostras hepáticas, porém as técnicas videolaparoscópicas permitiram acesso mais amplo ao fígado do que as técnicas percutâneas. A qualidade das amostras recuperadas com ambas as técnicas VL foram equivalentes, entretanto o uso da técnica de ressecção permitiu a colheita de amostras de maior peso, mas determinou a formação de aderências perihepáticas. Verificaram-se maior dificuldade na recuperação de fragmentos, maior oscilação do peso e menor qualidade das amostras obtidas com o uso das agulhas $\mathrm{M}$ do que com o das agulhas $\mathrm{T}$.

TERMOS DE INDEXAÇÃO: Biópsia hepática, videolaparoscopia, ultra-sonografia, ovinos.

\section{INTRODUÇÃO}

Entre os recursos auxiliares disponíveis para investigação ou estabelecimento do diagnóstico de doenças hepáticas em animais domésticos, encontram-se as provas bioquímicas, a radiologia e a ultra-sonografia (Radostits et al. 2002). Embora possam confirmar indícios de comprometimento do órgão e determinar a relativa severidade da doença, esses métodos raramente fornecem diagnósticos específicos (Bunch et al. 1985, Barr 1995). Neste contexto, o exame histopatológico obtido através de biópsias é essencial para o estabelecimento de diagnósticos e prognósticos mais precisos (Bunch et al. 1985). Além disso, a colheita de amostras seriadas de tecido é uma alternativa importante para a investigação histológica de doenças tóxicas (Colodel et al. 2000), para análise de substâncias em estudos de nutrição animal (Hidiroglou \& Ivan 1993), bem como para estudos que abordem a patogênese e a evolução das lesões hepáticas.

Na maior parte dos estudos sobre biópsias hepáticas em ovinos, os procedimentos foram conduzidos a partir de variações da técnica percutânea cega. Foram testados diferentes locais de punção e uma variedade de instrumentos e agulhas. Os resultados obtidos geralmente foram satisfatórios, com boa recuperação das amostras e mínimas complicações pós-operatórias (Harvey et al. 1984, Ferreira et al. 1996). Já as técnicas de biópsia hepática guiadas por ultra-sonografia (Lofstedt et al. 1988), ou assistidas por laparoscopia (Phillippo 1973, Hidirogolou \& Ivan 1993) foram pouco exploradas na espécie ovina, apesar da reconhecida precisão e segurança relacionadas com o seu uso. De qualquer forma, ainda que tenham sido obtidos resultados positivos com o uso de algumas técnicas e instrumentos diversos, não existem artigos dis- poníveis que comparem, de forma ampla, os efeitos das técnicas sobre os animais experimentais, nem que confrontem a representatividade e a qualidade das amostras obtidas através das diferentes técnicas.

Este trabalho foi desenvolvido com o objetivo de se compararem algumas técnicas de biópsias hepáticas, exeqüíveis em condições de campo, no que diz respeito à taxa de recuperação, qualidade e peso das amostras, repetibilidade dos procedimentos e ocorrência de complicações e efeitos adversos. Foram comparadas duas técnicas por videolaparoscopia e seis técnicas percutâneas, a partir do uso combinado de dois tipos de agulha com abordagens às cegas, guiadas por ultra-sonografia e por videolaparoscopia.

\section{MATERIAL E MÉTODOS}

\section{Animais}

Foram utilizadas oito borregas (quatro da raça Santa Inês e quatro Santa Inês X Texel), com idade entre seis meses e um ano e peso médio de $17,95 \mathrm{~kg} \pm 2,71$. Os animais, provenientes de pastagens diversas, foram alocados em baias coletivas no Hospital Veterinário da Escola de Veterinária da UFMG, alimentados com capim elefante, feno de Tifton e ração comercial com $18 \%$ de proteína bruta $(\mathrm{PB})$, vermifugados e pesados em intervalos semanais.

\section{Procedimento experimental}

Este trabalho foi aprovado pelo Comitê de Ética em Experimentação Animal, CETEA-UFMG sob protocolo no 200/06. Utilizou-se delineamento em quadrado latino (8X8), em que cada animal foi submetido, uma única vez, a todas as oito técnicas de biópsia hepática, executadas com intervalos consecutivos de uma semana. Em seis técnicas empregaram-se dois tipos de agulha (Menghini modificada e Tru-cut semi-automática) ${ }^{7}$ em biópsias percutâneas cegas, guiadas por ultra-sonografia e por videolaparoscopia. Os dois tipos de agulha foram reutilizados para cada técnica no decorrer do experimento. Para permitir o reaproveitamento das agulhas Tru-cut semi-automáticas foi aplicada cola a base de resina epóxi ${ }^{8}$ sobre a superfície de apoio da cânula externa da agulha. Nas outras duas técnicas, foram testados pinça laparoscópica de biópsia modelo Blakesley e o método de ressecção de fragmento hepático por videolaparoscopia. Em cada procedimento, as biópsias foram repetidas até se obter um fragmento de tecido com tamanho adequado para avaliação histológica.

O instrumental cirúrgico e as agulhas de biópsia percutâneas foram submetidos à desinfecção com solução de glutaraldeído a $2 \%{ }^{9}$ por 15 minutos e lavados em seguida com solução fisiológica estéril. Após jejum alimentar de 24 horas e sedação com cloridrato de xilazina a $2 \%{ }^{10}(0,1 \mathrm{mg} / \mathrm{kg})$, os animais eram contidos em decúbito lateral esquerdo para realização dos procedimentos de tricotomia e anti-sepsia do campo cirúrgico. No local da biópsia e nos pontos de introdução dos conjuntos

\footnotetext{
${ }^{7}$ Bloodline ${ }^{\circledR}$, Bloodline S.p.A., 12 Via Statale, Medolla, MO, Italia.

${ }^{8}$ Araldite ${ }^{\circledR}$, Brascola Ltda, Rua Brascola 222, São Bernardo do Campo, SP.

${ }^{9}$ Glutaron II ${ }^{\circledR}$, Indústria Farmacêutica Rioquímica Ltda, Av. Tarraf 2590/2600, Jardim Anice, São José do Rio Preto, SP.

${ }^{10}$ Rompun ${ }^{\circledR}$, Bayer S.A., Estrada Boa Esperança 650, Belford Roxo, RJ.
} 
trocarte/cânula foram infiltrados um volume de $5 \mathrm{ml}$ de solução cloridrato de lidocaína a $2 \%{ }^{11}$.

\section{Técnicas de biópsia hepática}

Biópsia hepática percutânea cega com agulha de Menghini modificada (MC). Uma agulha de Menghini modificada foi introduzida em sentido crânio-ventral, no $11^{\circ}$ espaço intercostal direito, no ponto de interseção com uma linha paralela à coluna vertebral, que partia da extremidade lateral da tuberosidade ilíaca (Fig.1). Após ultrapassar a parede torácica e a superfície do fígado, o êmbolo da seringa foi tracionado até acionar o mecanismo de trava da seringa. Em seguida, a agulha foi introduzida no parênquima hepático, por dois a três centímetros. Nesta etapa realizou-se um movimento sutil de recuo e avanço da ponta da agulha em sentido oblíquo. Findo este procedimento, procedeuse a retirada da amostra do interior da agulha.

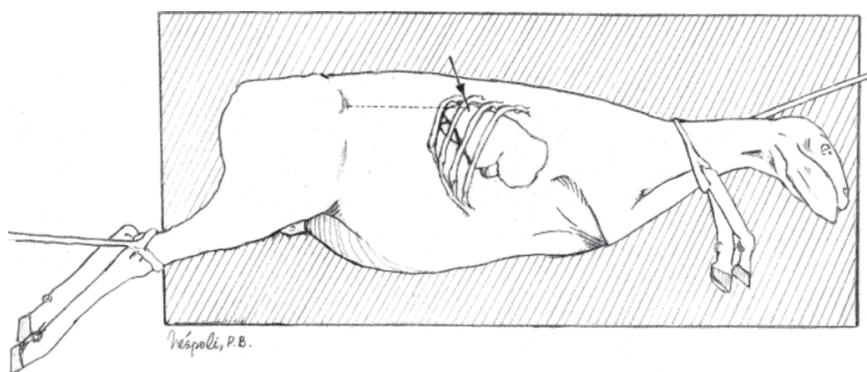

Fig.1. Posicionamento do animal e definição do local para realização das biópsias hepáticas percutâneas. Posicionamento: decúbito lateral esquerdo. Local: ponto de interseção do $11^{\circ}$ espaço intercostal com uma linha paralela à coluna vertebral, que parte da extremidade lateral da tuberosidade ilíaca. Agulha inserida nos sentidos medial e crânio-ventral (seta).

Biópsia hepática percutânea cega com agulha Tru-cut semi-automática (TC). Uma agulha Tru-cut semi-automática foi introduzida através de pequena incisão de pele $(0,3 \mathrm{~cm})$ no mesmo local descrito anteriormente. Uma vez que a ponta da agulha estava apoiada sobre a superfície hepática, toda a haste obturadora interna da agulha foi avançada no parênquima. Em seguida, através do disparo de dispositivo, a cânula externa da agulha avançava automaticamente sobre o entalhe da haste obturadora. Em seguida, a agulha Tru-cut semi-automática foi retirada para a manipulação do fragmento de tecido hepático.

Biópsia hepática percutânea guiada por ultra-sonografia com agulha de Menghini modificada (MU) e com agulha Trucut semi-automática (TU). Para a execução destas duas técnicas foram utilizados os mesmos procedimentos descritos anteriormente, porém a introdução e manipulação das agulhas de Menghini modificada (MU) e da Tru-cut semi-automática (TU) no fígado foram acompanhadas por inspeção indireta, através de imagens ultra-sonográficas. Foi utilizado para tanto, um aparelho de ultra-sonografia modelo Eureka AS-600 ${ }^{12}$, equipado com transdutor linear de $5 \mathrm{MHz}$, sem guia para a passagem da agulha de biópsia.

\footnotetext{
${ }^{11}$ Anestésico L®, Eurofarma Laboratórios Ltda, Av. Nações Unidas 22215, Jurubatuba, São Paulo, SP.

${ }^{12}$ Eureka AS-600®, Medison Co. Ltd, 1003 Daechi-dong Gangnamgu, Seoul, Korea.
}

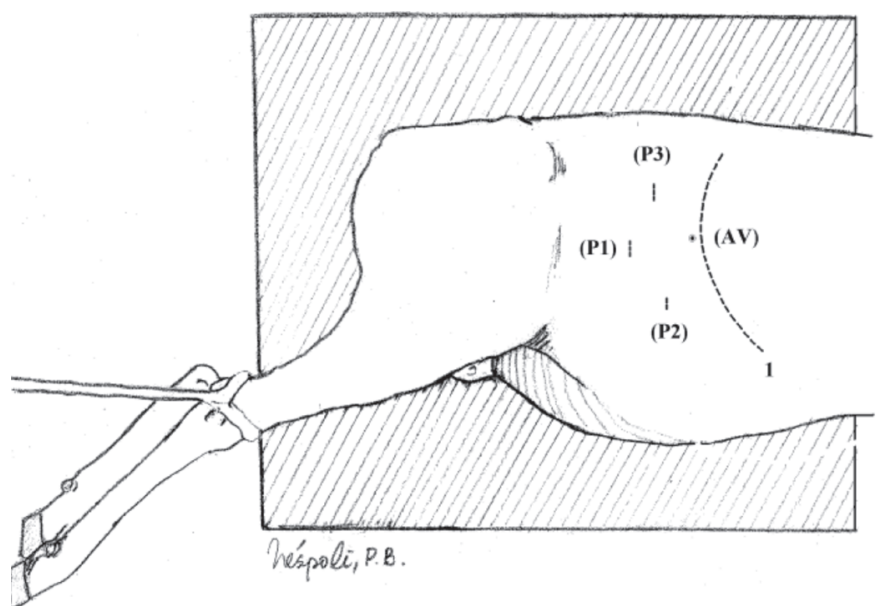

Fig.2. Local de estabelecimento dos portais para videolaparoscopia e para introdução da agulha de Veress. (1) Margem caudal do gradil costal. (AV) Local para introdução da aguIha de Veress. (P1) Local do primeiro portal para introdução do endoscópio. (P2 e P3) Segundo e terceiro portais para introdução de instrumentais.

Biópsia hepática por videolaparoscopia com pinça de Blakesley (PL). Após a desinfecção do material de videolaparoscopia $^{13}$ e preparação do animal, foi realizada a infiltração local com 5ml de lidocaína nos pontos de incisão P1 e P2 (Fig.2). Em seguida, a agulha de Veress foi introduzida na cavidade abdominal na porção dorsocranial do flanco direito, logo atrás da última costela. Através desta agulha, foi instalado o pneumoperitônio com $\mathrm{CO}_{2}$ a um fluxo de 0,5 litro/minuto com manutenção de pressão intra-abdominal constante em torno de $10 \mathrm{~mm}$ de $\mathrm{Hg}$. Foram realizadas duas incisões de pele nos pontos de infiltração anestésica para estabelecer, através da introdução de trocarteres de 11 e $6 \mathrm{~mm}$, os portais para o endoscópio (P1) e para a pinça de Blakesley (P2), respectivamente. Após a visualização da superfície hepática, a pinça de Blakesley foi introduzida na cavidade para retirada de um fragmento hepático sobre a margem ventral do lobo direito (Fig.3B). O sangramento resultante foi observado apenas no período imediato pós-biópsia. Na seqüência, o gás e todos os instrumentos foram retirados da cavidade e a pele suturada com fio cirúrgico mononailon $2.0^{14}$ em pontos simples interrompidos.

Biópsia hepática percutânea por videolaparoscopia com agulha de Menghini modificada (ML) e agulha Tru-cut semiautomática (TL). Para a execução destas duas técnicas se repetiram os mesmos procedimentos relativos à videolaparoscopia descritos para a técnica PL. Porém, a retirada de fragmento hepático foi mediada pelo uso de agulha de Menghini modificada na técnica ML e pela agulha Tru-cut semi-automática na técnica $T L$, de acordo com o procedimento descrito para as biópsias percutâneas (MC e TC, respectivamente) (Fig.3A).

Ressecção de fragmento hepático por videolaparoscopia (RL). Nesta técnica procedeu-se a triangulação com três portais para permitir o uso simultâneo de dois instrumentos laparoscópicos (Fig.2). Nos portais P1, P2 e P3 foram introduzidos o endoscópio, tesoura cirúrgica e pinça de dissecção,

\footnotetext{
${ }^{13}$ Karl Storz ${ }^{\circledR}$, Karl Storz GmbH \& Co. KG, Mittelstr.8, Tuttlingen, Baden-Württenberg, Alermanha.

${ }^{14}$ Shalon ${ }^{\circledR}$ suturas, Shalon Fios Cirúrgicos Ltda, Av. Hermógenes Coelho 3523, Alto da Boa Vista, São Luiz de Montes Belos, GO.
} 

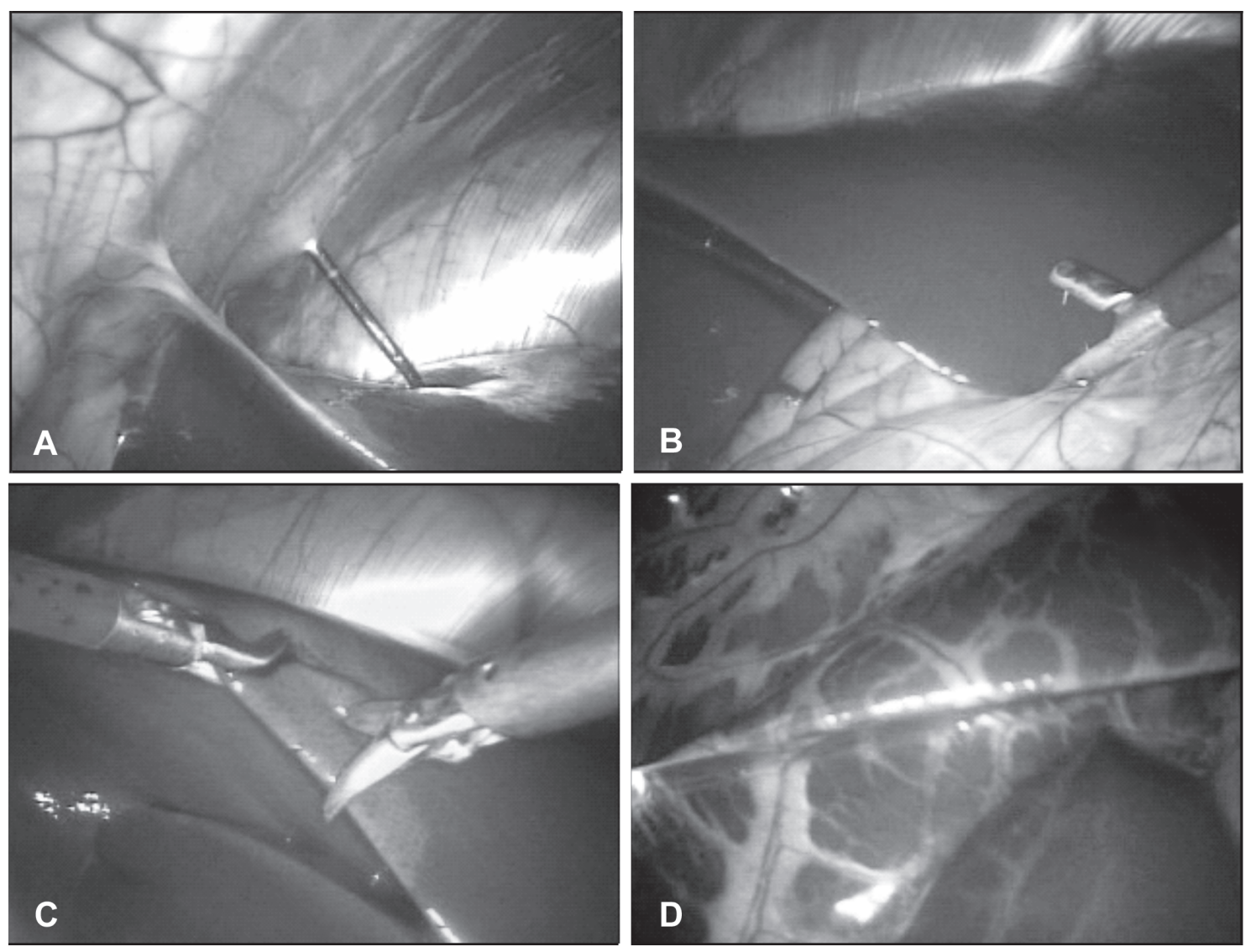

Fig.3. (A) Imagem endoscópica da introdução da agulha Tru-cut na cavidade abdominal e no parênquima hepático (TL). (B) Imagem endoscópica do posicionamento da pinça de Blakesley para apreensão da borda hepática (PL). (C) Imagem endoscópica da ressecção de fragmento hepático com tesoura de dissecção (RL). (D) Lâmina profunda do omento maior sobre a superfície do lobo direito do fígado.

respectivamente. Após a apreensão da margem ventral do órgão com a pinça, foi realizada uma incisão semi-circular da borda caudolateral do lobo direito com a tesoura (Fig.3C) e o fragmento resultante foi retirado através da cânula do portal 3.

\section{Processamento do material}

Todos os fragmentos hepáticos obtidos em cada procedimento, independente do número de tentativas, foram fixados em um único frasco com solução de formol a $10 \%$ por 48 horas, pesados (após secagem em papel filtro) e enviados para o Setor de Anatomia Patológica, Convênio Universidade Federal Rural do Rio de Janeiro/Embrapa. Esses fragmentos foram processados rotineiramente para estudo histológico e corados pela Hematoxilina e Eosina. As amostras foram avaliadas através da contagem dos espaços-porta e de veias centrolobulares, da qualidade da amostra, bem como pela identificação de artefatos (fragmentação da amostra, presença de hemorragia e de grupos de hepatócitos eosinofílicos).

\section{Estudo clínico e hematimetria}

Foram realizados exames clínicos diários dos animais, de acordo com a metodologia descrita por Stöber (1993). Após os procedimentos de biópsia, os animais foram medicados com dose única de oxitetraciclina ${ }^{15}$ e de flunixin meglumine ${ }^{16}$.

A hematimetria foi realizada através de contagem automática de células com o aparelho Abacus Junior Vet ${ }^{17}$ antes e du- rante o experimento, em intervalos semanais. As análises de gama glutamil transferase (GGT), aspartato aminotransferase (AST), albumina e proteína total foram realizadas um dia antes e três dias depois de cada procedimento através de espectrofotometria.

\section{Análise estatística}

Os dados relacionados com o peso das amostras, o número de tentativas despendidas, o número de veias centrolobulares e o número de espaços-porta foram submetidos à aplicação do teste não paramétrico de KruskalWallis. As ordenações médias foram comparadas utilizando-se o teste de Student-Newman-Keuls (SNK), através da aplicação do programa Bioestat 4.0 (Ayres et al. 2005).

\section{RESULTADOS}

Foram observadas alterações clínicas nos animais em 10 dos 64 procedimentos realizados. Na maioria dos casos,

\footnotetext{
15 Terramicina ${ }^{\circledR}$ LA, Laboratórios Pfizer Ltda, Av. Monteiro Lobato 2270, Guarulhos, SP.

${ }^{16}$ Flunamine ${ }^{\circledR}$, Bayer S.A., Estr. Boa Esperança 650, Belford Roxo, RJ.

${ }^{17}$ Abacus Junior Vet ${ }^{\circledR}$, Diatron MI Ltd, 58-59 Utca, Papírgyár, Budapest, Hungary.
} 
os achados se restringiram às elevações leves a moderadas da temperatura corporal e da freqüência cardíaca, nos primeiros dois dias após a realização das biópsias. Somente quatro, destes 10 animais, manifestaram alteração do estado geral, manifestos por apatia e inapetência, quatro apresentaram diminuição da freqüência e intensidade dos movimentos ruminais, um dor abdominal, um tremores musculares e um mucosas congestas. Ainda assim, os sinais clínicos foram de curta duração, com recuperação em dois ou três dias. Em todos estes quatro casos foram utilizadas agulhas de Menghini e em dois procedimentos o número de tentativas foi igual ou superior a duas.

O número de eritrócitos e os valores do hematócrito permaneceram dentro dos limites de referência $\left(9-15 \times 10^{6}\right.$ / $\mathrm{ml}$ e $27-45 \%$, respectivamente). Os valores da AST se mantiveram entre 100 e $150 \mathrm{UI} / \mathrm{l}$ e, na maioria das aferições, abaixo de $205 \mathrm{UI} / \mathrm{I}$, dentro dos limites de referência para a espécie ovina (60-280 UI/I) (Radostits et al. 2002).

A maior parte dos animais apresentou níveis séricos de GGT situados entre 30 e $60 \mathrm{UI} / \mathrm{I}$ dentro dos limites de referência (40-94 UI/I) (Smith 2002). Dois animais (39 e 109), entretanto, demonstraram valores superiores aos demais. Os níveis de GGT do animal 39 decresceram gradativamente, desde o valor de $107 \mathrm{UI} / \mathrm{l}$ até concentrações próximas dos demais animais. Já as concentrações de GGT do animal 109 oscilaram de forma ampla durante todo o experimento e em duas ocasiões foram superiores a $94 \mathrm{UI} / \mathrm{l}(98$ e $107 \mathrm{UI} / \mathrm{l})$.

Os valores médios da proteína total e da albumina situaram-se abaixo dos limites de referência ( 6 e 2,4g/dl, respectivamente) (Radostits et al. 2002) entre a 4ㄹ e a 6a semanas.

As lesões hepáticas relacionadas com as biópsias percutâneas restringiram-se a pequenas áreas circunscritas de fibrose com leve depressão da superfície hepática. O uso da pinça laparoscópica produziu áreas de fibrose um pouco mais extensas, com deformação e retração do bordo hepático. Por outro lado, as lesões associadas com a técnica de ressecção foram mais graves e abrangentes. Dos cinco animais avaliados durante o experimento, quatro apresentaram aderências do fígado com estruturas adjacentes como intestino delgado ou omento.

Entre as técnicas percutâneas auxiliadas por recursos de imagem, o uso da ultra-sonografia imprimiu maior agilidade ao procedimento e de forma menos invasiva do que através da videolaparoscopia. Em contrapartida, o emprego da videolaparoscopia garantiu acesso a toda a superfície parietal do fígado, inclusive de porções inacessíveis através da ultra-sonografia como o lobo esquerdo.

A principal dificuldade encontrada durante os procedimentos de videolaparoscopia foi a perfuração da bolsa omental. Após a introdução do endoscópio verificava-se uma face do omento sobre as vísceras abdominais (Fig. 3D), o que impedia a realização das técnicas de biópsia hepática por videolaparoscopia. Para contornar esta dificuldade o $\mathrm{CO}_{2}$ foi retirado da cavidade abdominal e todo o procedimento inicial da videolaparoscopia foi reiniciado, o que resultou em acréscimo considerável ao tempo cirúrgico.

A hemorragia observada durante os procedimentos de videolaparoscopia foi discreta e equivalente naquelas biópsias com agulhas de Menghini e Tru-cut. Apesar de ter havido um sangramento relativamente maior nas biópsias com a pinça laparoscópica, a hemorragia observada foi autolimitante. Por outro lado, a hemorragia verificada com a execução da técnica ressecção por videolaparoscopia foi mais acentuada, principalmente quando as secções se estenderam mais para o centro do órgão. Já naquelas secções mais localizadas na periferia do lobo direito o sangramento foi menos intenso.

Os resultados relacionados com os números de tentativas empregadas e o peso das amostras hepáticas obtidas estão dispostos nos Quadros 1 e 2. Os valores médios de veias centrolobulares e de espaço-portas das biópsias e os intervalos de variação estão disponíveis nos Quadros 3 e 4.

Quadro 1. Comparação do número médio de tentativas despendidas para obtenção de amostras representativas e do peso médio $(\mathrm{mg})$ dos fragmentos hepáticos de acordo com as técnicas de biópsia empregadas

\begin{tabular}{lcccccccc}
\hline & $\mathrm{MC}^{*}$ & $\mathrm{TC}$ & $\mathrm{MU}$ & $\mathrm{TU}$ & $\mathrm{ML}$ & $\mathrm{TL}$ & $\mathrm{PL}$ & $\mathrm{RL}$ \\
\hline Número de tentativas & $1,5^{\mathrm{ab}}$ & $1,125^{\mathrm{ab}}$ & $2,375^{\mathrm{b}}$ & $1^{\mathrm{a}}$ & $1,625^{\mathrm{ab}}$ & $1,125^{\mathrm{ab}}$ & $1,125^{\mathrm{ab}}$ & $1,25^{\mathrm{ab}}$ \\
Peso total & $48,5^{\mathrm{c}}$ & $32,2^{\mathrm{b}}$ & $52,2^{\mathrm{c}}$ & $26,2^{\mathrm{a}}$ & $61,6^{\mathrm{c}}$ & $30,9^{\mathrm{ab}}$ & $132,3^{\mathrm{d}}$ & $1362,4^{\mathrm{e}}$ \\
Peso/ tentativas & $41,0^{\mathrm{a}}$ & $29,4^{\mathrm{a}}$ & $29,8^{\mathrm{a}}$ & $26,2^{\mathrm{a}}$ & $45,9^{\mathrm{a}}$ & $27,8^{\mathrm{a}}$ & $124,5^{\mathrm{b}}$ & $1151,6^{\mathrm{c}}$
\end{tabular}

a Médias com letras iguais na mesma linha não diferem significativamente $(p<0,05)$. ${ }^{*} M C=$ Biópsia cega $x$ agulha de Menghini. TC $=$ Biópsia cega $x$ agulha Tru-cut. $M U=$ biópsia guiada por ultrasonografia $\times$ Menghini. TU = Biópsia guiada por ultra-sonografia $\times$ Tru-cut. ML = Biópsia por videolaparoscopia $\times$ Menghini. TL = Biópsia por videolaparoscopia $\times$ Tru-cut. PL = Biópsia com pinça laparoscópica. $\mathrm{RL}=$ Ressecção por videolaparoscopia.

Quadro 2. Intervalo de variação do peso das amostras $(\mathrm{mg})$ hepáticas obtidas com apenas uma tentativa

\begin{tabular}{|c|c|c|c|c|c|c|c|c|}
\hline & $\mathrm{MC}^{*}$ & $\mathrm{TC}$ & MU & TU & ML & TL & $\mathrm{PL}$ & $\mathrm{RL}$ \\
\hline Variação do peso & 31,7 a 74 & 23,3 a 34,6 & 22,9 a 70,5 & 23,1 a 30,8 & 37,2 a 88,5 & 22,4 a 34,1 & 96,1 a 260,8 & 599,7 a 1667,5 \\
\hline Biópsias com uma tentativa & 6 & 7 & 3 & 8 & 5 & 7 & 7 & 6 \\
\hline
\end{tabular}


Quadro 3. Comparação do número médio de veias centrolobulares (VCL) e de espaçosporta (EP) de acordo com a técnica de biópsia empregadas

\begin{tabular}{lcccccccc}
\hline & $M^{*}$ & TC & MU & TU & ML & TL & PL & RL \\
\hline$N^{\circ}$ total VC & $14,25^{\mathrm{ab}}$ & $13,25^{\mathrm{ab}}$ & $14,25^{\mathrm{ab}}$ & $11,625^{\mathrm{a}}$ & $19,5^{\mathrm{b}}$ & $13,625^{\mathrm{ab}}$ & $18,125^{\mathrm{ab}}$ & Incontáveis \\
№ VC/ tentativas & $12.084^{\mathrm{a}}$ & $12.13^{\mathrm{a}}$ & $8.67^{\mathrm{a}}$ & $11.625^{\mathrm{a}}$ & $14.938^{\mathrm{a}}$ & $12.688^{\mathrm{a}}$ & $17.25^{\mathrm{a}}$ & Incontáveis \\
№ total EP & $27,5^{\mathrm{abc}}$ & $22,5^{\mathrm{ab}}$ & $30^{\mathrm{bc}}$ & $21,75^{\mathrm{a}}$ & $34,25^{\mathrm{bc}}$ & $26,625^{\mathrm{abc}}$ & $42,625^{\mathrm{c}}$ & Incontáveis \\
№ $\mathrm{NP} /$ tentativas & $23.25^{\mathrm{a}}$ & $20.688^{\mathrm{a}}$ & $17.423^{\mathrm{a}}$ & $21.75^{\mathrm{a}}$ & $25.688^{\text {ab }}$ & $24.063^{\mathrm{a}}$ & $38.438^{\mathrm{b}}$ & Incontáveis \\
\hline
\end{tabular}

a Médias com letras iguais na mesma linha não diferem significativamente $(p<0,05)$. ${ }^{*} \mathrm{MC}=$ Biópsia cega $x$ agulha de Menghini. TC = Biópsia cega $\times$ agulha Tru-cut. um = biópsia guiada por ultra-sonografia $\times$ Menghini. TU = Biópsia guiada por ultra-sonografia $\times$ Tru-cut. $\mathrm{ML}=$ Biópsia por videolaparoscopia $\times$ Menghini. TL = Biópsia por videolaparoscopia $\times$ Tru-cut. $\mathrm{PL}=$ Biópsia com pinça laparoscópica. $\mathrm{RL}=$ Ressecção por videolaparoscopia.

Quadro 4. Intervalo de variação do número de veias centrolobulares e espaços-porta das amostras obtidas com apenas uma tentativa

\begin{tabular}{lccccccc}
\hline & MC $^{*}$ & TC & MU & TU & ML & TL & PL \\
\hline VCL & 8 a 23 & 6 a 18 & 11 a 16 & 6 a 19 & 12 a 27 & 9 a 18 & 10 a 49 \\
EP & 18 a 38 & 8 a 34 & 19 a 33 & 13 a 28 & 19 a 53 & 17 a 33 & 23 a 78
\end{tabular}

* $\mathrm{MC}=$ Biópsia cega $\mathrm{X}$ agulha de Menghini. $\mathrm{TC}$ = Biópsia cega $\mathrm{x}$ agulha Tru-cut. um = biópsia guiada por ultra-sonografia $\times$ Menghini. $T U=$ Biópsia guiada por ultra-sonografia $x$ Tru-cut. ML = Biópsia por videolaparoscopia $\times$ Menghini. TL = Biópsia por videolaparoscopia $\times$ Tru-cut. $\mathrm{PL}=$ Biópsia com pinça laparoscópica. $\mathrm{RL}=$ Ressecção por videolaparoscopia.

A técnica de ressecção por videolaparoscopia foi a única que permitiu a obtenção de amostras com qualidade máxima em todas as repetições, porém com resultados equivalentes aos das técnicas PL e TL. Entre as técnicas percutâneas, a qualidade das amostras obtidas com agulhas Tru-cut foi melhor do que aquelas obtidas com agulhas de Menghini.

No quesito fragmentação parcial das amostras, as técnicas PL e RL foram relacionadas com melhor desempenho frente às demais técnicas. Aquelas conduzidas com agulhas Tru-cut foram equivalentes entre si e associadas com graus menores de fragmentação das amostras do que aquelas realizadas com agulhas de Menghini.

Houve relativa uniformidade quanto à presença de grupos de hepatócitos eosinofílicos entre as amostras com todas as técnicas de biópsia empregadas e maiores gradações de hemorragia relacionadas com as amostras obtidas com a técnica RL.

\section{DISCUSSÃO E CONCLUSÕES}

\section{Alterações propostas às técnicas originais}

O posicionamento do animal e o local escolhido para realização das biópsias percutâneas (no $11^{\circ}$ espaço intercostal, na altura da tuberosidade ilíaca) garantiram grande agilidade aos procedimentos de biópsias percutâneas cegas e taxa de recuperação de amostras, similar às demais técnicas percutâneas guiadas por ultra-sonografia e por videolaparoscopia. Na literatura consultada, não há relato de emprego de metodologia semelhante à utilizada no presente trabalho. Os locais de biópsia descritos variam desde a interseção do $8^{\circ}$ espaço intercostal (Pearson \& Craig 1980) até o início da fossa paralombar direita
(Hogan et al. 1971) com pontos localizados a uma distância fixa da coluna vertebral (Hogan et al. 1971, Harvey et al. 1984, Ferreira et al. 1996) ou com linhas de referências anatômicas (Dick 1944, Scherer et al. 2000).

\section{Emprego auxiliar das técnicas de ultra-sonografia e videolaparoscopia}

Embora tenha sido demonstrada a grande versatilidade da ultra-sonografia no monitoramento das biópsias percutâneas, deve-se ponderar que a realização das biópsias se aplica somente à área de projeção ultrasonográfica do órgão, que nos ruminantes se estende principalmente do $9^{\circ}$ ao $12^{\circ}$ espaço intercostal (Braun 1996). Desta forma, este método permite acesso amplo ao lobo direito e caudado, mas restringe o alcance do lobo quadrado e esquerdo, que ficam sobrepostos pelos pulmões em praticamente toda a sua extensão.

Em contrapartida, as biópsias conduzidas com auxílio da videolaparoscopia podem ser realizadas em qualquer porção da superfície parietal do fígado, inclusive do lobo esquerdo. $\mathrm{O}$ acesso amplo às biópsias é particularmente útil para se aferir a concentração in vivo de substâncias em diferentes porções do fígado, procedimentos que antes só eram possíveis após o sacrifício dos animais (Anderson et al. 1962, Hogan 1971). A técnica tem sido testada por Hidiroglou \& Ivan (1993), em biópsias seriadas dos lobos direito, quadrado e esquerdo sem a ocorrência de complicações relevantes. Além disso, a videolaparoscopia propicia o controle visual do procedimento, útil para realização de biópsias em lesões localizadas e superficiais do parênquima hepático (Kerwin 1995), para a colheita de grande quantidade de tecido de forma segura (Silva et al. 2002) e para a identificação precoce (Kerwin 1995) e correção imediata de complicações inerentes à técnica (Costa et al. 2005).

\section{Avaliação das amostras}

Houve maior dificuldade em se obter amostras com as agulhas de Menghini do que com as Tru-cut. Esta menor eficiência de métodos aspirativos frente aos sistemas de guilhotina já havia sido apontada em estudo similar de biópsias percutâneas em bovinos (Buckley et al. 1986). No presente estudo, as falhas no mecanismo de sucção das agulhas de Menghini foram, aparentemente, responsáveis pela menor eficiência destes instrumentos. É provável que o fator determinante para isso tenha sido a 
reutilização da agulha, um instrumento descartável, com maior parte dos componentes de plástico.

Já o uso das agulhas Tru-cut foi associado às melhores taxas de recuperação das amostras, com médias de tentativas e desvios padrões menores. Além da relatada eficiência do método de guilhotina (Buckley et al. 1986), outro fator que parece ter contribuído para este melhor resultado foi o mecanismo de avanço semi-automático da cânula da agulha, que reduz a necessidade de repetição da técnica por garantir a recuperação de amostras maiores do que as obtidas com agulhas Tru-cut manuais (Hoope et al. 1986). Diferente do que ocorreu com as aguIhas Menghini, a reutilização não afetou o funcionamento das agulhas Tru-cut, fato já apontado em trabalho correspondente (Kerwin 1995).

A quantidade de tecido recuperada com as agulhas de Menghini foi comparativamente maior do que com as aguIhas Tru-cut, muito embora, tenha havido grande oscilação entre os valores obtidos. Por outro lado, com agulhas Tru-cut garantiram-se amostras com pesos mais uniformes, porém mais limitados que variaram em média de 26-29mg (Quadro 1). Os resultados equivalentes entre as biopsias percutâneas realizadas com o mesmo tipo de agulha indicam ainda que o peso das amostras não sofreu interferência significativa do método utilizado para guiar os procedimentos de biópsia, como a ultra-sonografia ou a videolaparoscopia.

Os pesos médios das amostras obtidos com agulhas Tru-cut foram discretamente superiores aos observados em outros estudos realizados em ovinos (Harvey et al. 1984, Ferreira et al. 1996). Em contrapartida, os pesos obtidos com agulhas de Menghini foram muito inferiores aos 800-1.200mg relatados por Braga et al. (1985).

As demais biópsias com abordagem videolaparoscópica propiciaram a obtenção de amostras com pesos bastante elevados. A técnica de ressecção $(\mathrm{RL})$ apresentou o maior valor médio, com peso de $1.150 \mathrm{mg}$, bastante superior a todos os demais tratamentos, e com o uso da pinça laparoscópica $(\mathrm{PL})$, obteve-se amostras com peso médio de 124mg (Quadro 1). O peso médio das amostras adquiridas com a técnica PL foi inferior ao relatado por Hidiroglou \& Ivan (1993) e o da técnica RL foram comparáveis às técnicas aspirativas realizadas em bovinos com conjuntos trocarte/cânula de grosso calibre (Seghetti \& Marsh 1953, Bone 1954, Erwin et al. 1956, Amorim et al. 2003).

O número de espaços-porta representou melhor a quantidade de tecido obtido com as biópsias do que a contagem de veias centrolobulares. Apesar da marcante diferença da quantidade de tecido recuperado com o uso da pinça laparoscópica quando comparado às biópsias percutâneas, o número de veias centrolobulares foi equivalente entre esses tipos de biópsia. Por outro lado, houve diferenças estatísticas entre o número de espaços-porta quando se efetivou a mesma comparação. A análise desses dados indica que o tipo de contagem realizado (vasos íntegros e não íntegros) produziu efeito que superestimou a representatividade da contagem das veias centrolobulares sobre o tamanho das amostras das biópsias percutâneas. Apesar de terem sido incluídas estruturas seccionadas na contagem do número de espaçosporta, os números médios relacionados com as técnicas percutâneas observados neste experimento foram bem superiores àqueles relatados por outros autores (Tostes \& Bandarra 2002, Vasanjee et al. 2006).

Com base nos dados disponíveis verifica-se ainda que todas as técnicas de biópsia hepática estudadas proporcionaram amostras adequadas para avaliação histopatológica, já que alguns estudos apontam que são necessários pelo menos quatro (Tostes \& Bandarra 2002, Costa et al. 2005) ou seis espaços-porta (Vasenjee et al. 2006) para que as amostras hepáticas sejam assim consideradas.

Embora tenha havido compressão da amostra durante a realização das técnicas de ressecção por videolaparoscopia, o grande volume de tecido obtido garantiu à técnica a melhor avaliação na qualidade da amostra e baixo grau de fragmentação parcial. De maneira similar, a pressão sofrida pela amostra durante a execução da técnica com pinça laparoscópica também não interferiu negativamente no resultado desses dois parâmetros. Entre as técnicas percutâneas, os melhores resultados foram obtidos com aquelas realizadas com agulhas Tru-cut, provavelmente, pelo mecanismo de corte destas agulhas, que aparentemente exerce menor efeito sobre a amostra do que o método aspirativo. Além disso, os resultados comparativamente melhores destas agulhas já eram esperados, uma vez que tais dispositivos semi-automáticos têm sido relacionados com amostras de alta qualidade (FinnBodner \& Hathcock 1993, Kerwin 1995), com menor fragmentação da amostra, com bordos mais bem definidos e menor formação de artefatos por compressão do que as agulhas com mecanismo totalmente manual (Finn-Bodner \& Hathcock 1993).

\section{Complicações causadas pelas técnicas}

As lesões hepáticas observadas com o uso das técnicas percutâneas com agulhas de Menghini ou Tru-cut foram mínimas e, por esta razão, essas técnicas são recomendadas em situações que demandam repetição de biópsias. Da mesma forma, apesar de produzir lesões mais extensas do que as biópsias percutâneas, a técnica PL não produziu aderências ou complicações maiores e também pode ser preconizada para procedimentos seriados. De fato, tanto as biópsias hepáticas percutâneas (Harvey et al. 1984) como as por laparoscopia (Hidiroglou \& Ivan 1993) têm sido realizadas de forma repetida em ovinos sem qualquer dificuldade ou complicação. Já a técnica de ressecção não é propícia para estudos que exijam repetição das biópsias, uma vez que determinou a ocorrência de um alto percentual de aderências nos animais examinados, provavelmente, em virtude do maior sangramento observado. Desta forma, a retirada de fragmentos menores poderia minimizar este efeito, bem como o uso de eletro-cauterização da ferida cirúrgica (Costa et al. 2005), ou o emprego do instrumental "harmonic scalpel" que 
secciona e cauteriza o tecido simultaneamente (Vasenjee et al. 2006).

\section{Alterações clínicas e laboratoriais associadas aos pro- cedimentos}

A realização das biópsias hepáticas seriadas pode resultar em alterações clínicas, entretanto, mesmo com o uso de agulhas de Menghini, os distúrbios observados foram leves, transitórios e de pequeno significado para a saúde dos animais. Fato comprovado pelo ganho de peso observado nas primeiras cinco semanas e estabilização na fase final do experimento. Estes resultados estão de acordo, pelo menos em parte, com os estudos de biópsia hepática em ovinos onde não foram observadas alterações clínicas (Harvey et al. 1984, Hidiroglou \& Ivan 1993) ou modificações no ganho de peso dos animais (Donald et al. 1984, Ferreira et al. 1996).

Não foram verificadas alterações hematológicas nos animais no decorrer do experimento, conforme constatado em outros estudos semelhantes (Harvey et al. 1984, Amorim et al. 2003). Da mesma forma, os níveis de AST sofreram pequenas elevações dentro dos limites de referência para a espécie (60-280UI/I) (Radostits et al. 2002) de forma similar ao observado em experimentos conduzidos em ovinos (Harvey et al. 1984) e bovinos (Braga et al. 1985).

As oscilações dos níveis de GGT observadas em dois animais ultrapassaram apenas, de forma discreta, os valores de referência para a espécie (40-94 UI/I) (Smith 2002). No primeiro caso, o animal recuperava-se de um quadro de fotossensibilização hepatógena causada pela ingestão de Brachiaria decumbens, comumente associada a aumentos de GGT (Lemos et al. 1996), e no outro caso, as elevações da enzima ocorreram após a 1aㅡ, $2^{\underline{a}}$, $4^{\text {a }}$ e $7^{\text {a }}$ biópsias. Tais elevações, entretanto, não coincidiram, necessariamente, com manifestações clínicas ou incrementos dos níveis de AST.

\section{REFERÊNCIAS}

Amorim R.M., Borges A.S., Kuchembuck M.R.G., Takahira R.K. \& Alencar N.X. 2003. Bioquímica sérica e hemograma de bovinos antes e após a técnica de biópsia hepática. Ciência Rural 33(3):519-523.

Anderson T.A., Taylor R.E., Diven R.H., Hubbert F. \& Hale W.H. 1962. Reability of the liver biopsy technique for estimating hepatic vitamin. Am. J. Anim. Sci. 21:369-372.

Ayres M., Ayres M.J., Ayres D.L. \& Santos A.A.S. 2005. Bioestat: aplicações estatísticas nas áreas das ciências biomédicas. Mamirauá, Belém, PA. Disponível em http://www.mamiraua.org.br. Acesso em 25 de setembro de 2007.

Barr F. 1995. Percutaneous biopsy of abdominal organs under ultrasound guidance. J. Small Anim. Pract. 36:105-113.

Bone J.F. 1954. A technic for aspiration liver biopsy in dairy cattle. North Am. Vet. 35:747-752.

Braga M.M., Castilhos L.M.L. \& Santos M.N. 1985. Biópsia hepática em bovinos: proposta de nova técnica. Ciência Rural 15(1):79-88.

Braun U. 1996. Ultrasonographic examination of the liver and gall bladder in cows: normal findings. Comp. Cont. Educ. Pract. Vet. 18(2):s61-s73.

Buckley W.T., Eigendorf G.K. \& Dorward W.J. 1986. A liver biopsy intrument for large animals. Can. J. Anim. Sci. 66:1137-1140.

Bunch S.E., Polak D.M. \& Hornbuckle W.E. 1985. A modified laparoscopic approach for liver biopsy in dogs. J. Am. Vet. Med. Assoc. 187(10):1032-1035.

Colodel E.M., Driemeier D. \& Pilati C. 2000. Intoxicação experimental pelos frutos de Xanthium cavanillesii (Asteraceae) em bovinos. Pesq. Vet. Bras. 20(1):31-38.

Costa P.R.S., Lopes M.A.F., Costa M.C. \& Lima Júnior L.O. 2005. Biopsia hepática videolaparoscópica em cães. Revta Ceres 52(303):763-770.

Dick A.T. 1944. Aspiration biopsy of the liver in sheep. Aust. Vet. J. 20:298-303.

Donald G.E., Paul D.R. \& Langlands J.P. 1984. Liver biopsy as a technique for assessing copper status of sheep. Aust. Vet. J. 61(4):121122.

Erwin E.S., Dyer I.A., Meyer T.O. \& Scott K.W. 1956. Uses of aspiration biopsy technique. J. Anim. Sci. 15:428-434.

Ferreira A.V., Van der Merwe H.J. \& Slippers S.C. 1996. A technique for obtaining liver biopsies from mature sheep. Small Rumin. Res. 22:89-92.

Finn-Bodner S.T. \& Hathcock J.T. 1993. Image-guided percutaneous needle biopsy: Ultrasound, computed tomography, and magnetic resonance imaging. Semin. Vet. Med. Surg. Small Anim. 8:258-278.

Harvey R.B., Lovering S.L., Bailey E.M. \& Norman J.O. 1984. The influence of multiple liver biopsies on hematologic and serum biochemical values of sheep. Cornell Vet. 74:322-330.

Hidiroglou M. \& Ivan M. 1993. Liver biopsy in sheep. Vet. Res. 24:260265.

Hogan K.G., Money D.F.L. \& Walker R.S. 1971. The distribution of copper in the liver of pigs and sheep and its effect on the value of chemical analyses made on biopsy samples. N.Z. J. Agric. Res. 14:132-141.

Hoope F.E., Hager D.A., Poulos P.W., Ekman S. \& Lindgren P.G. 1986. Comparison of manual and automatic ultrasound guided biopsy techniques. Vet. Radiol. 27:99-101.

Kerwin S.C. 1995. Hepatic aspiration and biopsy techniques. Vet. Clin. North Am. Small Anim. Pract. 25(2):275-303.

Lemos R.A.A., Ferreira L.C.L., Silva S.M., Nakazato L. \& Salvador S.C. 1996. Fotossensibilização e colangiopatia associada a cristais em ovinos em pastagem com Brachiaria decumbens. Ciência Rural 26(1):109-113.

Lofstedt J., Schelling S., Stowater J. \& Morris E. 1988. Antemortem diagnosis of hepatic adenocarcinoma in a ewe. J. Am. Vet. Med. Assoc. 193(12):1537-1538.

Pearson E.G. \& Craig A.M. 1980. The diagnosis of liver disease in equine and food animals. Mod. Vet. Pract. 63(3):233-238.

Phillippo M. 1973. A technique for liver biopsy in sheep. J. Agric. Sci. 80:313-314.

Radostits O.M., Gay C.C., Blood D.C. \& Hinchcliff K.W. 2002. Clínica Veterinária: Um tratado de doenças dos bovinos, ovinos, suínos, caprinos e eqüinos. 9ª ed. Guanabara Koogan, Rio de Janeiro, p.1645-165.

Scherer P.O., Pile E.A., Serra-Freire N.M. \& Schäffer G.V. 2000. Modificação da técnica de punção-biópsia hepática para pequenos ruminantes. Revta Bras. Ciênc. Vet. 7(1):55-57.

Seghetti L. \& Marsh H. 1953. Liver biopsy in cattle. Am. J. Vet. Res. 14:9-11.

Silva L.C.L.C., Stopiglia A.J. \& Fantoni D.T. 2002. Técnica de biópsia hepática em eqüinos por laparoscopia. Ciência Rural 32(3):459-465.

Smith P.S. 2002. Large Animal Internal Medicine. $3^{\text {rd }}$ ed. Mosby, St Louis, p.1735.

Stöber M. 1993. Identificação, anamnese, regras básicas da técnica de exame clínico geral, p.166-225. In: Rosenberger G. (Ed.), Exame Clínico dos Bovinos. $3^{\mathrm{a}}$ ed. Guanabara Koogan, Rio de Janeiro.

Tostes R.A. \& Bandarra E.P. 2002. Biopsia hepática em cães: relação entre qualidade da amostra e grau de conclusão do diagnóstico. Arq. Bras. Med. Vet. Zootec. 54(5):468-472.

Vasanjee S.C., Bubenik L.J., Hosgood G. \& Bauer R. 2006. Evaluation of hemorrhage, sample size, and collateral damage for five hepatic biopsy methods in dogs. Vet. Surg. 35:86-93. 